\title{
Conflict in Pedestrian Networks
}

\author{
Jia Wang, Zena Wood, and Mike Worboys \\ University of Greenwich, Old Royal Naval College, 30 Park Row, London SE10 9LS \\ $\{$ J.Wang, Z.Wood, M. Worboys\} @greenwich.ac.uk
}

\begin{abstract}
Encouraging pedestrian activity is increasingly recognised as beneficial for public health, the environment and the economy. As our cities become more crowded, there is a need for urban planners to take into account more explicitly pedestrian needs. The term that is now in use is that a city should be 'walkable'. For route planning, whereas much attention has been given to shortest path, in distance or time, much less attention has been paid to flow levels and the difficulties they pose on the route. This paper considers problems posed by conflicting paths, for example cross-traffic. We use network centrality measures to make a first estimate of differing levels of conflict posed at the network nodes. We take special note of the role of collective motion in determining network usage. A small case study illustrates the method.
\end{abstract}

Keywords: walkability, network centrality, collective movement, conflict

\section{Introduction}

Encouraging pedestrian activity is increasingly recognised as beneficial for public health, the environment and the economy. Walkability is the measure of how friendly an environment is to walking [1] and has become an important dimension in urban planning. A walkable city provides an accessible walking environment that encourages more pedestrian activity, thus providing the benefits noted in the first sentence.

As part of a walkability quality measure, existing research has developed several methods that focus on crowding. Most methods are concerned with the physical properties of pedestrian flows (speed and volume) and walking areas (size). (See, for example, [2]). A certain level of crowding can make a street inviting and lively. However, there is a level at which crowding leads to congestion with conflicting motion creating an uncomfortable environment with poor walkability [3]. When navigating an over-crowded environment, pedestrians need to constantly change their trajectories and speed to avoid conflicting motion. This can be stressful and can constrain walking $[4,5]$.

Our concern is to identify hot-spots where congestion or conflict provide obstacles to pedestrian movement. Within this paper the pedestrian system of walkways and intersections is modelled as an undirected graph, with walkways as edges and intersections as nodes. A method is presented where each node is 
assigned a resistance which indicates how much 'energy' a pedestrian might need to navigate through that node. These resistances are then used to compute the betweenness-centrality for each node to make a first estimate of differing levels of conflict posed at the network nodes. Conflict at a node would occur when paths cross and pedestrians have to navigate through potential collisions. We assume that the amount of conflict at a node is proportional to the number of crossing paths through the node. Figure 1 illustrates how a pedestrian environment could be represented as a graph. The blue paths within (a) show the different paths that a pedestrian may take through the node. The node is then expanded to a hyper-node (b) to clearly show the crossing paths and, therefore, where conflict will occur.

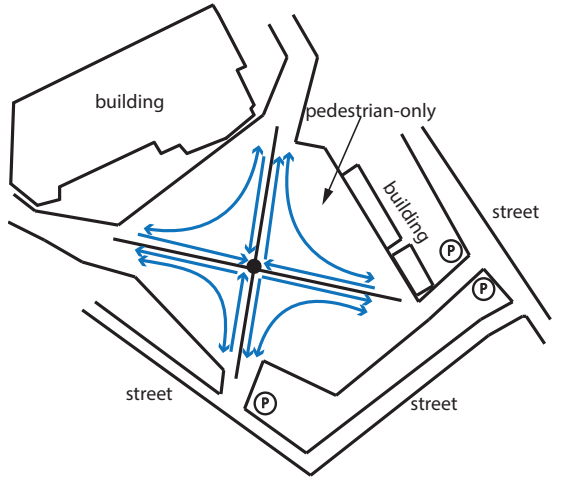

a. node

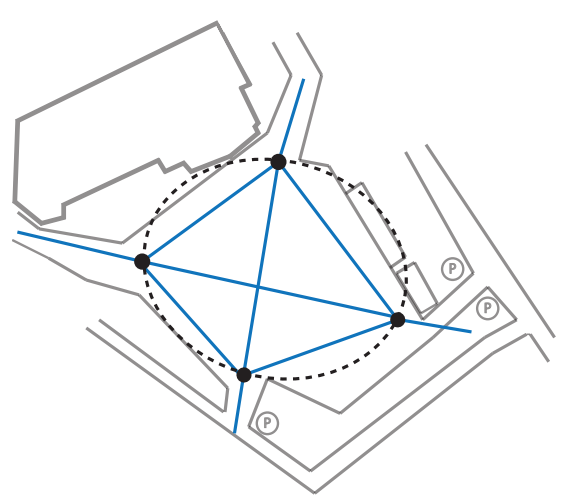

b. hyper-node

Fig. 1. a) Pedestrian system represented as a graph with conflicting motion occurring at the intersection. b) The intersection is expanded as a hyper-node (dotted circle) to highlight the conflict

Analysing the common flows through the network is one way to identify hotspots where conflict is more likely to occur. A collective is a group of individuals that for some reason we wish to refer to as a single entity [6]. When considering the walkability of an area it is often more useful to consider the data at the level of the collectives and not the level of the individuals. The evolving collective behaviour within a large group of individuals is often what an analyst requires [7]. Considering a large number of entities at the level of the collectives instead of the level of the individuals can also be computationally more efficient. Pedestrians can be grouped according to their travel purposes [8], origin and destination points [9], or shared movement characteristics $[6,10]$. This paper compares the proposed method when considering random flows versus collective motion of the network.

The paper continues in Section 2 by introducing related work on walkability, network centrality and collective movement. Section 3 describes the proposed method which is then applied in Section 4 to a network of popular pub crawls 
through Greenwich, London. The results of the implementation are discussed in Section 5. Future work is outlined in Section 6 and Section 7 concludes the whole paper.

\section{Related Work}

This section reviews related work from three areas: walkability measurement, focussing on congestion and conflicting movement, network centrality measures and collective movement.

\subsection{Walkability measurement}

The term walkability originates from the transportation literature and has been used widely within urban planning, transportation and public health research to assess how environmental factors affect pedestrian walking behaviour [11-14]. The concept of walkability is defined as the quality of the walking environment perceived by pedestrians who live, shop and visit there $[15,16]$, or as "the measure of the extent to which the public realm provides for movement and other activity on foot in ways that are both efficient and enjoyable" [17, p.4]. The operational definitions of walkability are provided at both macro and micro level, and are referenced to several components that can be observed and quantified (or qualified) on different spatial scales [16]. On a macro-level (or neighbourhood level), the walkability measure is usually based upon the assessment of street pattern, land use diversity and housing density: A connected and accessible street pattern contributes to better walkability; increased land use diversity and housing density reduce the trip distance to amenities and increase pedestrian safety $[12,14]$. Frank et al. [11] proposed a walkability index including four components: net residential density, retail floor area ratio, intersection density and land use mix. The walkability value is then calculated based on the values of these four components. On a micro-level (street or pedestrian level), walkability is measured by developing and scoring multiple environmental indicators related to a local route. The Pedestrian Environmental Factor index defines and quantifies walkability by scoring the four indicators as ease of crossing, sidewalk continuity, local street characteristics and topography [18]. The pedestrian Level-of-Service (LOS) quantifies walkability by relating it to pedestrian facilities with regard to pedestrian flow [2]. Other indices quantifying walkability on the micro-level include the Transit Friendliness Factor [19], the Walking Suitability Assessment $[20]$ and the Irvine-Minnesota Inventory [21, 22].

Besides the above-mentioned quantitative methods, qualitative measures relating to subjective pedestrian perception have also been used to define and measure walkability. For example, Ewing and Handy [23] pointed out that using physical components only to measure walkability may not provide details relating to the walking experience in a particular environment. Ewing and Handy qualified walkability based on the ratings from a panel of urban design experts and 
then concluded five perceptual elements that determined walkability as imageability, visual enclosure, human scale, transparency, and complexity [23]. This approach was further developed to operationalise eight subjective perceptual qualities as imageability, enclosure, human scale, transparency, complexity, legibility, linkage and coherence in the context of commercial streets [24].

In general, pedestrians prefer to avoid contact with others except when overcrowding cannot be avoided. Existing studies of urban design and pedestrian behaviour proposed several approaches on defining and measuring crowding. LOS defined different levels to measure the quality of pedestrian flows based on both volumes and sidewalk or crosswalk area [2]. Pedestrian Comfort Levels classified the level of comfort on the basis of the level of crowding experienced on the street, and the pedestrian crowding was measured in pedestrians per metre of clear footway width per minute [25]. Gehl [26] concluded that 13 people per meter per minute of footway was the maximum at which a comfortable level of quality can be delivered for footpath. Behavioural experiments involved personal space preferences into account and proposed the concept of minimum personal occupancy in dealing conflicting movement [4]. Other human aspects included attaining normal walking speeds to avoid conflicts with other pedestrians [4]. Existing research also pointed out that conflicting movements frequently happened in areas such as bus stops, tube exits, shopping centres and crossings [25].

\subsection{Collective movement}

When trying to improve an urban area it is important to try to satisfy as many people as possible and, therefore, you need to identify the needs of the "collective population' [27]. Identifying areas or locations of common interest is a common goal in movement pattern analysis [28]. The locations of interest could be origin and destination points [9] or those that are frequently visited [27]. Analysing origin and destination points within travel behaviour can help identify the need for new facilities, such as bus stops. Groups that share similar movement patterns could also be of interest. Such information could be obtained from a spatiotemporal dataset via clustering, aggregation and similarity calculations [28].

Focusing on general trends and collectives can be computationally more efficient especially when dealing with increasingly large datasets. Andrienko and Andrienko [9] use data aggregation to visually analyse traffic data within Milan. Collective movement is defined as a function that relates the set of moving entities over the set of possible time moments and positions in space. The data can be viewed in two ways: trajectory-oriented view and traffic-oriented view. The former view groups the trajectories and the latter considers the possible 'traffic situations'. The view adopted will depend on the goals of the analyst. Aggregation methods are suggested that group the trajectories according to origin and destination points, the points visited on a journey and the similarity of routes. This approach was taken further by Wang et al. [29] in an analysis of Eulerian and Lagrangian perspectives on motion.

Many types of collective can be considered in pedestrian movement [30]. These phenomena often arise due to the interactions and behaviour characteris- 
tics of the pedestrians, particularly self-organisation. Schadschneider et. al [30] use a cellular automaton (CA) to simulate these interactions to obtain the observed collectives. Four types of collective phenomenon are considered: jamming, lane formation, oscillations and panic. Jamming occurs when a blockage is encountered by pedestrians due to a lack of space or contradictory flow. Counterflows can result in the formation of lanes and observed self-organisation. When there is a blockage and an individual manages to make their way through, it becomes easier for other individuals to follow them. This continues until someone makes their way through in the opposite direction. This repeated pattern is what the term oscillation refers to. Panics occur where the movement is counterintuitive as a result of some situation (e.g., the wish for faster motion actually results in a slower moving crowd).

Andrienko and Andrienko [7] refer to two types of collective behaviour that an analyst may wish to focus on: Momentary Collective Behaviour (MCB) and Dynamic Collective Behaviour (DCB). The former focuses on a set of entities at a particular moment of time; the latter on multiple entities over a given temporal period. Four categories are identified that could influence the movement of entities including the activities and properties of the moving entities (e.g., how they move). Different patterns are specified that may be of interest and relevance to analysing DCB one of which is co-location in space (i.e., when the paths followed by the observed entities contains at least some of the same positions). The positions visited can be further analysed according to any variation occurring in the order of the locations visited. This movement pattern could be of relevance to a network, and identifying hotspots of conflict, if each node was considered as a location of interest.

\subsection{Network centrality}

Freeman [31], proposed a collection of centrality measures that indicate degrees to which nodes have significance in a network. Let $G$ be a graph, where $N$ is the number of nodes and $E$ is the number of edges. The betweenness centrality of a node of $G$ gives a measure of how much the node is an intermediate point on paths in $G$. To be more precise, the betweenness centrality of node $n$ is proportional to the number of shortest paths in $G$ that pass through $n$. Formally:

$$
B_{n}=\frac{1}{(N-1)(N-2)} \frac{\alpha_{n}}{\beta_{n}}
$$

where $B_{n}$ is the betweenness centrality of node $n, \alpha_{n}$ is the number of shortest paths between any two nodes (except $n$ ) in the graph passing through $n$, and $\beta_{n}$ is the number of shortest paths between any two nodes (except $n$ ) in the graph.

There is a body of research that has applied betweenness and other centrality measures to networks of urban streets. Varoudis et al., [32] evaluated the angular betweenness measure of space syntax of urban streets implemented by two different methods, Tasos and depthmapX. The depthmapX method is based on the cognitive-search-agent with pedestrian walking constraints. The Tasos 
method is based on mathematical shortest path without the pedestrian walking constraints used by the depthmapX method. The evaluation showed that these two methods offered similar results in terms of pedestrian movement but he Tasos method was more computationally efficient.

Kazerani and Winter identified in [33] the issues (e.g., no travel behaviour and temporal constraints) of using betweenness centrality in predicting traffic flow in reality and suggest some minor amendments to the classic centrality measure. The paper proposed a modified betweenness centrality method, which reflected locations of origins and destinations of recorded trips. Compared to the classic method, the proposed method was better in predicting "actual traffic counts over given time intervals" [33, p. 8].

Crucitti et al., [34,35] studied centrality in urban streets for 18 different world cities and developed a comparative analysis of different centrality measures of urban streets. The centrality measures were based on a new approach called multiple centrality assessment (MCA) developed by Porta et al., for the centrality analysis in geographic systems [36]. MCA was based on primal graphs, a set of centrality indices as well as a fully metric computation of distances. The results of Crucitti's study indicated that the centrality measures with the four indices (closeness, betweenness, straightness and information) allowed extended visualisation and characterisation of city structures.

Based on the MCA centrality analysis, Porta's group used the northern Italian city Bologna as study area to investigate "how much higher street centrality statistically determines a higher presence of activities (shops and services)" [37]. The result highlighted a strong correlation between street centrality (particularly betweenness centrality) and locations of shops and services at the neighbourhood scale. Following the same MCA method, Produit's Master thesis [38, p. 96] reports a new GIS tool that is able to create three indexes of network density estimation of activities, network density of edges with population made from centralities and diversity of activities along the network. The centrality indexes (closeness, betweenness and straightness) were computed at both global and local level to characterise the shape of network.

While most centrality studies focused on degree, closeness, betweenness and eigenvector measures to determine who occupied critical positions in the network, a rarely answered question was about the correlation of the four centrality measures [39]. Valente et al., empirically examined the correlation among the four centrality measures and found that they were strongly correlated. Their study also revealed the association of network properties such as density and reciprocity to the correlation of different centrality measures.

\section{Method}

There are problems with directly applying centrality measures to congestion and conflict in urban flow. Firstly, existing methods take little or no account of spatial and temporal variation in flows in the network. For example, direct application of the betweenness centrality measure to indicate those nodes which 
are more likely to be congested would require the assumption that flows are uniform in the network and that no one path is more used than another. Our work will generalise betweenness centrality to account for preferred paths followed by collective motion against a background of random noise in network flow.

The second problem is that no account is taken of conflict at nodes where paths cross and so pedestrians must negotiate through potential collisions. We make a simplifying assumption that the amount of conflict at a node is dependent upon the number of crossing paths through that node.

\subsection{Rotation graph and crossing paths}

In order to generalise previous constructions, it is necessary to take into account the embedding of graph $G$ in Euclidean space. We assume that $G$ is embedded in a surface and that each node is specified by a pair of coordinates. The embedding gives us information not only about the positions of nodes but also the positions and orientations of the edges. If we consider a single node $n$ of $G$, then those edges that are incident with $n$ are incident in a unique rotational cycle about $n$. This is shown by the example in figure 2 , where the five edges $e_{1}, e_{2}, \ldots, e_{5}$ are incident with $n$ in the specific cyclic order $\left(e_{1}, e_{2}, e_{5}, e_{4}, e_{3}\right)$. If graph $G$ has the property that each of its nodes is equipped with a rotation cycle of edges around it, then the graph is termed a rotation graph [40, p. 741].

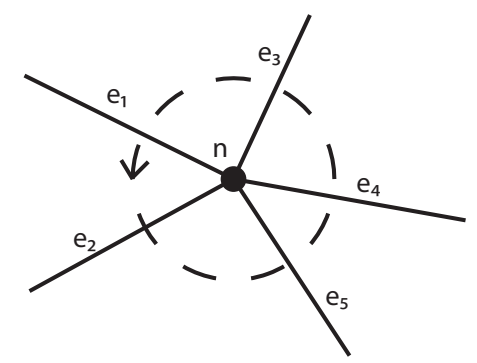

Fig. 2. Cycle of edges $e_{1}, e_{2}, \ldots, e_{5}$ around node $n$

Because the pedestrian network is modelled here as a graph embedded in Euclidean space, it is automatically a rotation graph. We can use the extra information provided by the cycles to give a better approximation of the energy required to traverse a path on the route taking into account conflicts due to crossing paths.

For example, consider again figure 2. In travelling through the node along edges $e_{1} e_{5}$, say, the traveller would potentially have to cross the paths of pedestrians on routes $e_{2}-e_{3}$ and $e_{2}-e_{4}$. (This is not taking into account direction of travel). Depending on the volume of traffic on the latter two routes, our traveller will meet more or less amounts of conflict. So, assuming we know the amounts 
of flow on the different paths, we can calculate the amount of resistance that each node on the path might add to the journey. We use this resistance to modify the travel distance between nodes to get a more accurate assessment of the betweenness coefficients.

In the general case, where a node has arbitrary degree. If the route through the node passes through edges $e$ and $f$, then the number of crossing paths is $i * j$ where $i$ and $j$ are the number of edges strictly between $e$ and $f$ in the cycle of edges, counting clockwise and anti-clockwise, respectively.

\subsection{Generalising betweenness}

As has been said, the betweenness centrality measure of a graph was originally conceived as a purely topological measure, not taking account of the graph embedding. However, as it involves shortest path computations, it is easy to extend to the case where path is defined between nodes using Euclidean distances along edges. Our contribution is to take into account node resistance to a path resulting paths that cross the path of interest. In the extreme case, we can neglect edge lengths and focus entirely on node resistances. As a very simple example, consider the network shown in figure 3 . Suppose we wish to calculate the path length $A D$. Then we need to take into account the crossing flows $\phi_{F C}, \phi_{E C}, \phi_{F B}, \phi_{E B}$. Just how much these cross-currents should contribute to the path length depends on the flow quantities and the level of disruption they course. However, once this has been calibrated, we can perform a Dijkstra-type computation for shortest paths, and from this calculate the betweenness centrality measures for nodes.

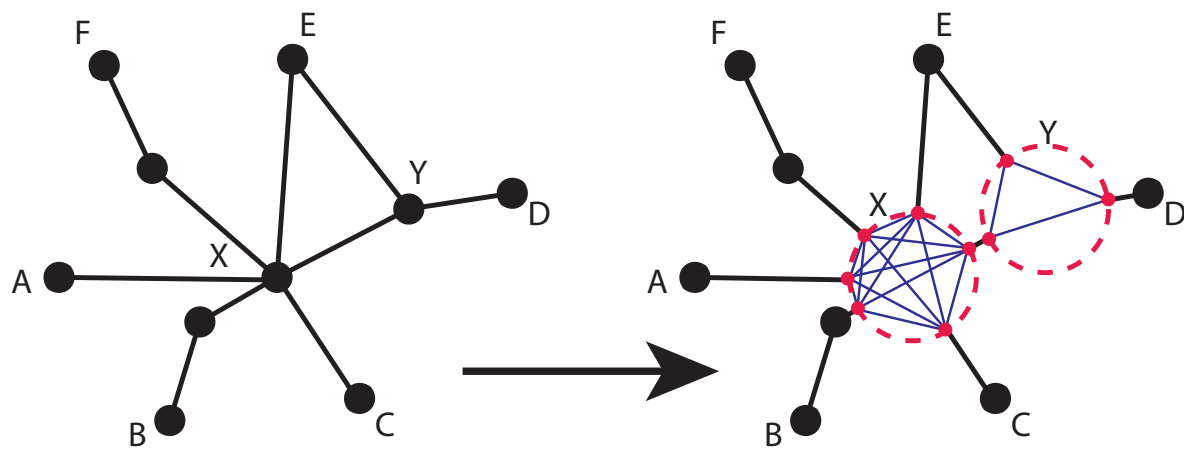

Fig. 3. Small network example

It is then interesting to compare the betweenness measures in three cases:

1. The traditional case with edge lengths calculated from geographic distances between nodes;

2. The case where edge lengths are ignored but node resistances determine path lengths. This leads to two sub-cases: 
(a) A random collection of flows is assumed through the network so all crossings are assumed to impart equal resistance

(b) Flows through the network arise from collectives passing through it. In this case, crossings are weighted by the flows through them.

In the next section we work through a case study showing how these constructions can be applied to a specific instance.

\section{Movement Data Analysis}

Two datasets have been used to demonstrate the proposed method both of which relate to pubs in Greenwich. Although neither dataset records the known movements of multiple individuals, we can demonstrate how the method could be used to identify conflict at nodes and show why collective movement should be considered.

\subsection{The Case Study}

A dataset containing the locations of 28 pubs within Greenwich has been used to produce the pedestrian network. The underlying road network has been used to form the network shown in figure 4 . Two pubs are considered connected if it is possible to travel from one to the other without coming across a third. Each node, depicted by a red circle, represents a pub and each edge, depicted by a black line, a connection between them.

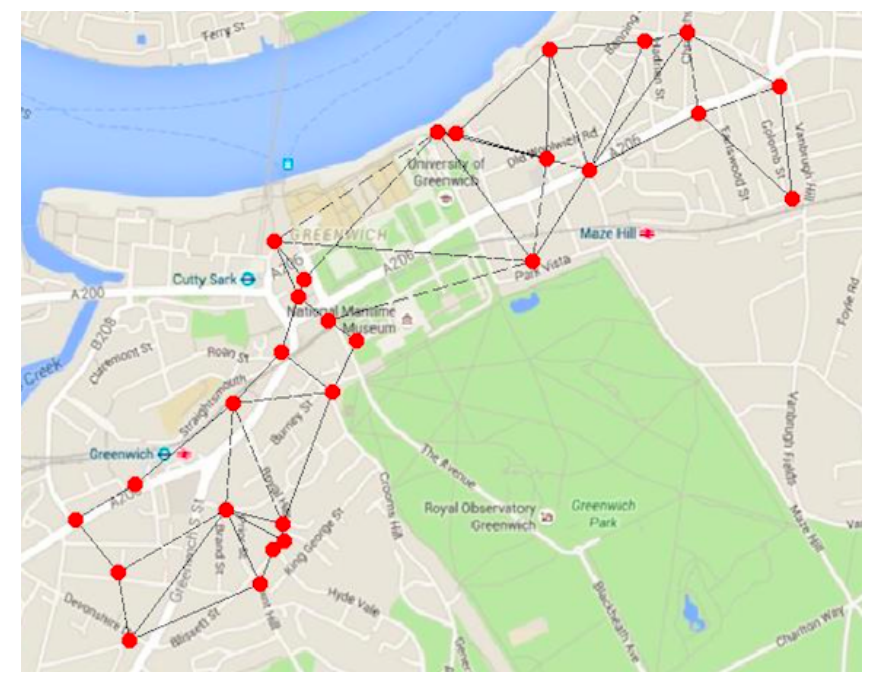

Fig. 4. The network of 28 pubs in Greenwich overlaid on a Google map 
Known pub crawls within Greenwich have been identified to model the collective movement within the network (figure 5). A pub crawl typically involves a group of individuals visiting a collection of pubs and having a specified number of beverages in each of them. Although unrealistic, it is assumed that collectives do not spend a significant amount of time at each pub and pass quickly through them. This allows the idea of pedestrians passing through nodes to be simulated and the need to navigate conflict. Ten pub crawls have been identified to model 10 collectives moving around the network. Dijkstra's algorithm has been used to calculate the shortest path between two consecutive pubs on a particular crawl that are not joined via an edge in the network.

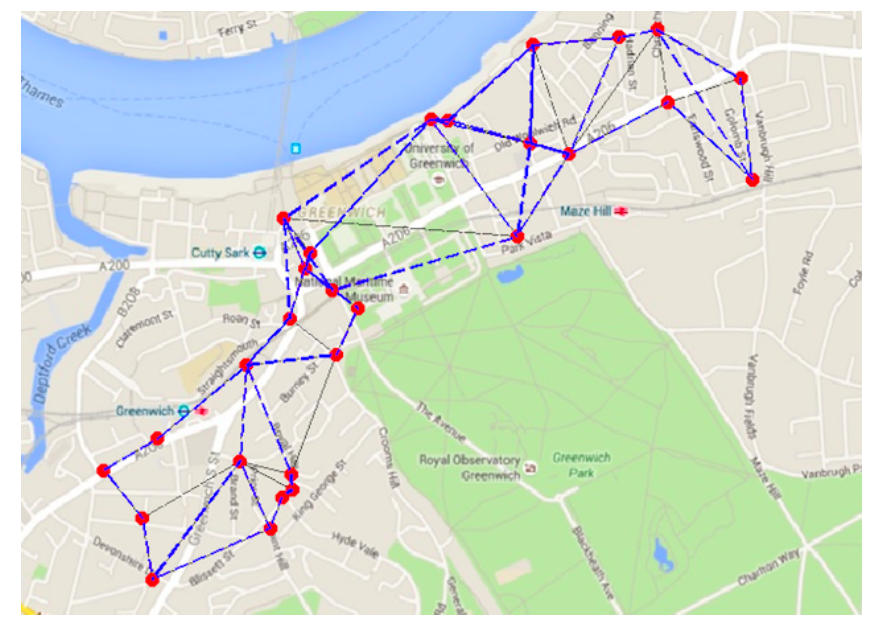

Fig. 5. The 10 pub crawls within Greenwich overlaid on a Google map

\subsection{Implementation}

The method has been implemented using Matlab. The program reads in two .csv files: one that stores the locations of each pub, recorded by their longitude and latitude values; and, one that records the order of pubs visited on each of the 10 pub crawls. The edges of the network are identified manually using a map of the underlying road network and stored using an adjacency matrix. If there is an edge connecting nodes $n_{i}$ and $n_{j}$ a 1 is placed in position (i, $\mathrm{j}$ ) of the matrix, otherwise the value will be 0 . A second adjacency matrix is computed that stores the geographic distance between each pair of connected nodes. For each node all possible paths that run through it are identified and, for each path, the number of cross flows calculated. The cross-flow values are combined to produce a value for the node's overall resistance.

When only considering geographic distance to calculate shortest path each edge is weighted with the distance between the two pubs that it connects. The 
weighting on each edge, when only considering resistance, is the combined value of the resistance contributed by the two nodes that it connects. For example, in figure 2, assuming a random collection of flows so all crossings impart equal resistance, the node would have a resistance of 10 . Since the node connects five edges the node would contribute a resistance of two to each of the five edges. The remaining weight of the edges would be the relevant resistance value from the other node that it connects. When considering collective motion on the network the node resistance is recalculated. Instead of considering all possible paths through a node only the paths that are used by the collectives are considered.

A second method has been adopted to distribute the node resistance between the edges when considering collective motion. This method distributes the node resistance proportionally between connecting edges according to usage. For example, figure 6 shows the usage of the collectives on the edges passing through a node (node $A$ ) within the network. Ten collectives pass through the node but the edges are not used equally. The node has a resistance value of two. Proportionally distributing this value amongst the connected edges would contribute a resistance value of 0.6 to edges $\phi_{A C}, \phi_{A D}$ and $\phi_{A E} ; \phi_{A B}$ would gain a resistance value of 0.1. The remaining weighting of the edges would come from the other node that they connect. This second distribution method has been included for comparison to see the affect that it has.

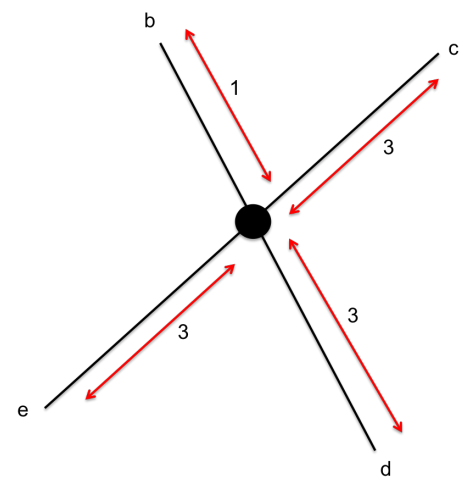

Fig. 6. An example of a node on the path of 10 collectives

Once the weights for each edge have been obtained, either using geographic distance or node resistance, the betweenness centrality of each node is calculated. For each of the 28 nodes in the network, an implementation of Dijkstra's algorithm is used to identify the number of shortest paths between each pair of nodes, excluding the current node being considering $(n)$, that pass through the node. The values are normalised by dividing by the possible number of shortest paths between two nodes in the network (not considering the current node $n$ ). 


\subsection{Results}

Figures 7 to 10 show the results of applying the proposed method to the sample dataset. In each figure a heat scale is used where red indicates the highest centrality value and blue the lowest. The same scale has been used for each figure to allow direct comparison of the results.

Figure 7 shows the betweenness centrality value for each node when only considering the geographic distance to calculate shortest path length. Figure 8 shows the betweenness centrality values when using node resistance to calculate shortest path length. A random collection of flows is assumed through the network so all crossings impart equal resistance. Figures 9 and 10 show the betweenness centrality of each node when considering collective motion using node resistance to calculate shortest path length. Within figure 9 node resistance is distributed equally amongst the connecting edges; figure 10 shows the results of proportional distribution of node resistance.

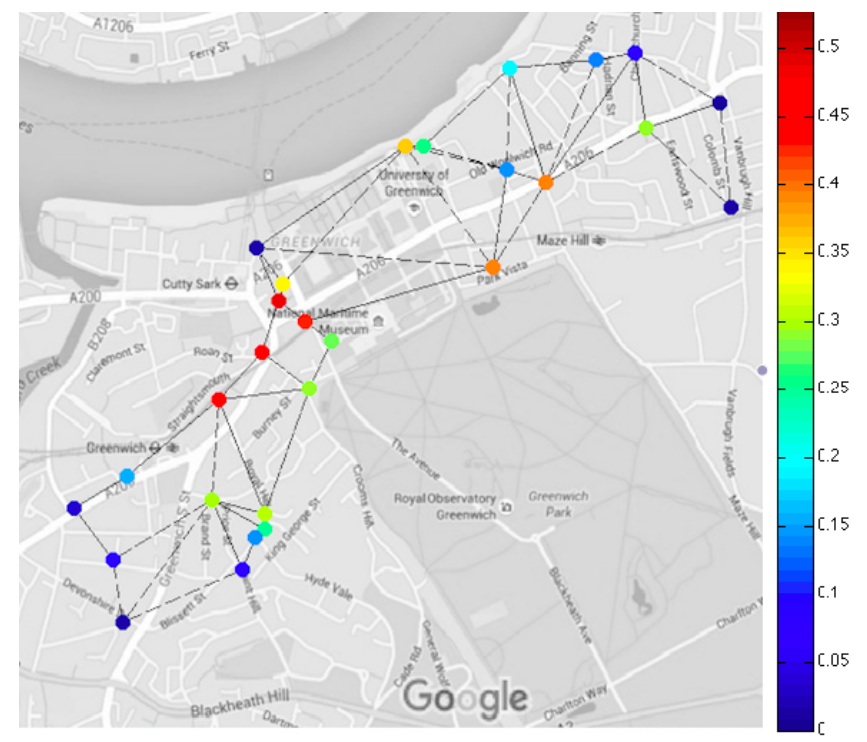

Fig. 7. The betweenness centrality of each node shown with a heat scale (red indicates the highest centrality value and blue the lowest) when edges are weighted according to distance

\section{Discussion}

The application of the proposed method to a sample dataset has shown that a different set of nodes within the network is identified as key (i.e., coloured red) when considering resistance and collective motion compared with general, 


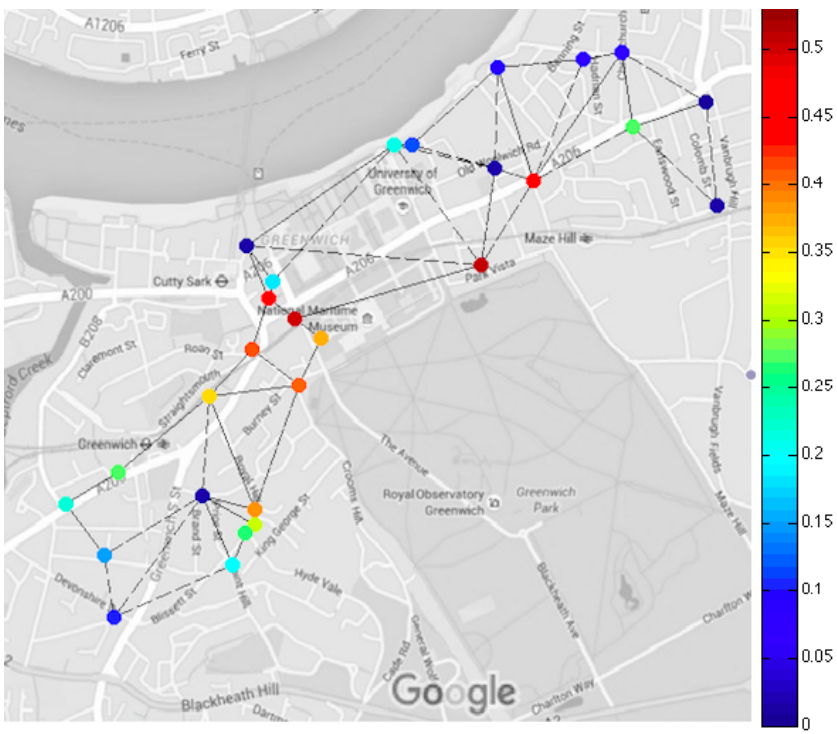

Fig. 8. The betweenness centrality of each node shown with a heat scale (red indicates the highest centrality value and blue the lowest) when edges are weighted according to node resistance assuming all crossings impart equal resistance

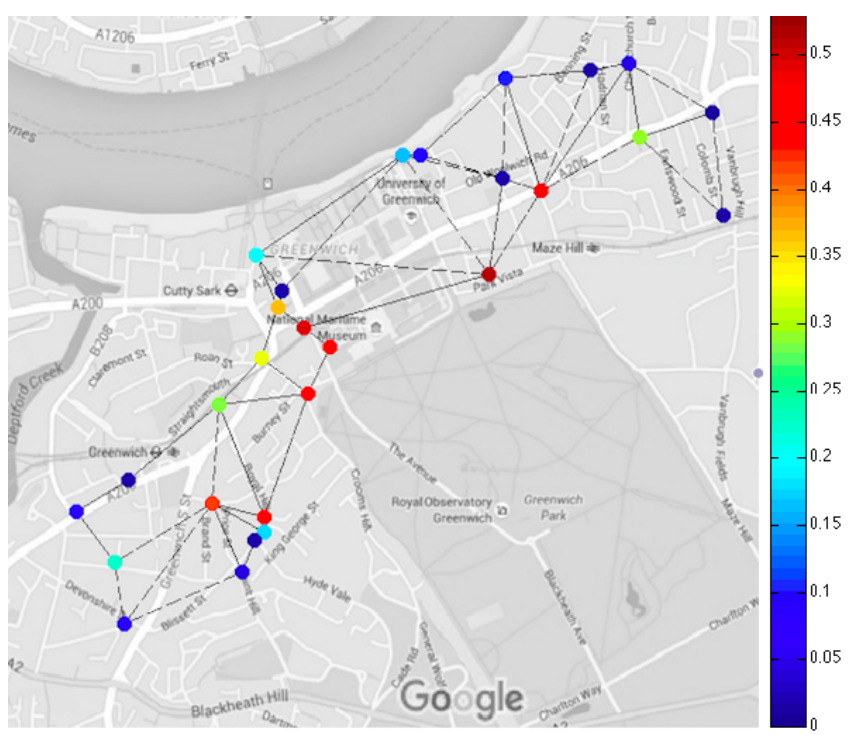

Fig. 9. The betweenness centrality of each node shown with a heat scale (red indicates the highest centrality value and blue the lowest) considering collective motion with node resistance distributed equally amongst connecting edges 


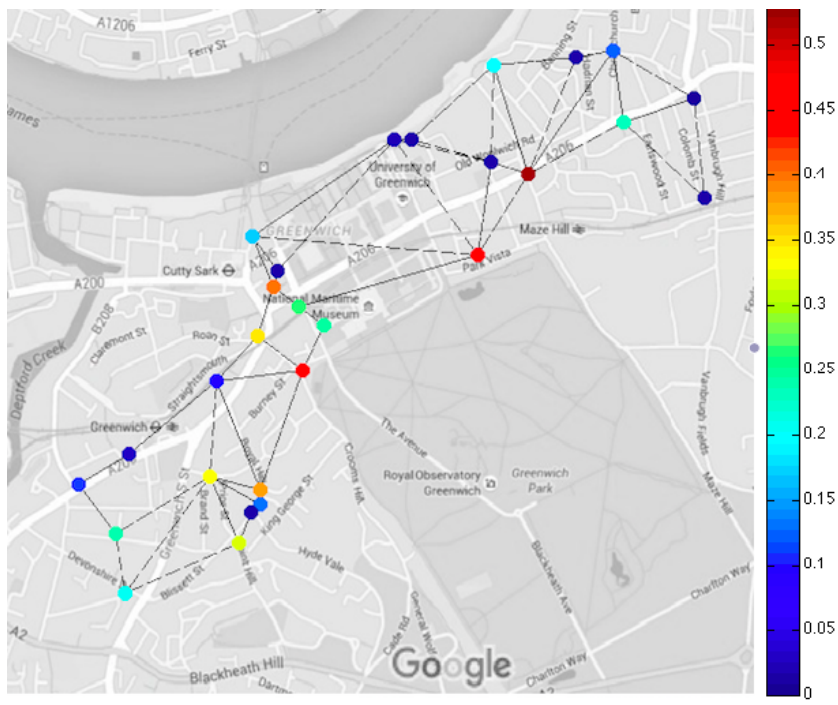

Fig. 10. The betweenness centrality of each node shown with a heat scale (red indicates the highest centrality value and blue the lowest) considering collective motion with proportionally distributed node resistance

random motion. This section discusses the results and aspects that have been highlighted by the analysis for further consideration.

In figure 7, where geographic distance was used to calculate shortest path, four nodes are shown in red and thirteen are in a shade of blue. The highlighted nodes are, as expected, the ones central within the network. The majority of the blue nodes lie on the outside edges of the network. When considering node resistance with a random flow on the network, assuming all crossings impart equal resistance, (figure 8 ) four nodes are again highlighted but these are not the same as though highlighted when considering geographic distance alone. Some nodes that were previously coloured yellow (mid-to high betweenness centrality measures) in figure 7 are now coloured blue. The nodes shown as having the highest centrality measures within figure 8 can still be considered as quite central within the network but not all outer edges have the lower centrality measures. Figures 9 and 10, where collective motion is considered, again show a different set of highlighted nodes. By allowing the key paths (i.e., the collective movement) to be considered, some of the nodes that were previously considered to be a point where conflict is likely to occur are now seen to be of little concern in figure 9 . It is also possible to quickly identify the main hotspots where there is likely to be conflict. Where the node resistances have been distributed equally between connecting edges (figure 9), there are many more nodes highlighted blue (i.e., with low betweenness centrality measures). However, there are some nodes, previously considered of low importance, that are now coloured in red. The spread of red nodes across the network is larger. Figure 10 shows what happens when 
node resistance is spread proportionally amongst connecting edges. A smaller number of nodes are highlighted as ones where conflict is likely to occur.

The dataset that we have used is only a sample and the numbers within each collective have not been taken into consideration. Future work will need to be undertaken to extend the method to take account of the flows within the network. This information could then be used to consider a network where an event is occurring such as a carnival, protest or major sporting event. In addition to showing where walkability may be affected, emergency services and event planners could also use the results of our method to identify where they may focus their resources during the event.

The dataset does not include any temporal information. It is likely that flows within the network will vary over time. Different collectives may only exist at specific times, which would affect the identified hotspots. For example, commuters travel at peak times. The method should be extended to show the temporal variation in the betweenness values of each node. The dataset to which it is applied also would need the necessary temporal information.

\section{Future Work}

Betweenness centrality is just one of several measures that might be used as an indicator of importance of a node in a network. Application and consideration of other measures, such as 'information centrality', are currently being investigated. Two methods were used to distribute the node resistance around the connecting edges. Both methods highlighted different sets of key nodes but further analysis is required to see which is the most meaningful and efficient when used in a larger network.

Similar to the walkability measure described in Section 2, resistance in a particular walking environment can be measured in two ways. One is to use the physical components of the walking space (e.g., capacities of intersections and pathways) in addition to network flows to decide different levels of resistance. The second approach is similar to Ewing and Handy's work [23] in which qualifies resistance based on subjective qualities to determine resistance from the human perspective. This is an area for future work.

The graph used within the analysis is a simplification of the underlying road network. Further analysis should be carried out that takes into account more detail of the real-world network. Although based on real locations and common paths, the dataset in this paper could be considered synthetic. Work has begun in identifying a dataset that has the recorded movements of a large number of pedestrians in a mapped environment. From this the collectives will be identified using existing methods (e.g., those described in [28]).

\section{Conclusion}

This paper has presented research on issues arising from conflicting motion in networks. Although many of the methods are generalisable to any transportation 
system, we have focused on pedestrian networks. Conflict arises at network nodes when paths cross, and a reasonable assumption made in this paper is that the more potential for paths to cross, both in terms of the number of possible routes through the node but also in terms of the amount of flow, the more potential for conflict there arises. Collective motion has an important influence on network flows. A key methodology adopted in this work is to use network centrality and, in particular, betweenness centrality to estimate the nodes in the network with the most potential for conflict. In traditional network centrality approaches, path weights are measured by length, either spatial or temporal. A key contribution here is to measure path weight by 'resistances' that conflicting paths induce. This resistance is determined by flows along conflicting paths, which in turn is determined by collective motions through the network.

In our case study of routes through pubs in Greenwich, the pubs were the nodes and the network was the collection of possible paths from one pub to the next. For the sake of this experiment, we took the extreme position of only considering conflict in the weighting of paths, and so distances were neglected. Betweenness centrality of the nodes was computed in two cases:

- Flow through the network was considered to be random.

- Flow through the network was determined by collective motion induced by the pub crawls.

The results clearly demonstrated the expected results that collective motion through a network strongly influenced the resistance of nodes to paths, and hence altered which nodes became key, as measured by betweenness centrality.

\section{Acknowledgements}

This study was supported by the University of Greenwich Faculty of Architecture, Computing and Humanities Research \& Enterprise Fund through the project "Spatial Informatics for the Dynamic Smart City".

\section{References}

1. City of Fort Collins, Colorado. (2011). Pedestrian Plan. http://www.fcgov.com/transportationplanning/pedplan.php

2. Gallin, N. (2001). Quantifying pedestrian friendliness-guidelines for assessing pedestrian level of service. Road \& Transport Research, 10(1), 47.

3. Karl Brierley (2013). The effects of pedestrian delay and overcrowding on our streets $\&$ the rationale for shorter blocks and through blocks links. A report prepared for the city of Melbourne.

4. Fruin, J. (1992). Designing for pedestrians. Public Transportation United States.

5. Handy, S. (2005). Critical assessment of the literature on the relationships among transportation, land use, and physical activity. Transportation Research Board and the Institute of Medicine Committee on Physical Activity, Health, Transportation, and Land Use. Resource paper for TRB Special Report, 282. 
6. Wood, Z. \& Galton, A. (2009). A taxonomy of collective phenomena. Applied Ontology, 4, (3-4), 267-292.

7. Andrienko, G. \& Andrienko, N. (2007). Extracting Patterns of Individual Movement Behaviour from a Massive Collection of Tracked Positions. Workshop on Behaviour Modelling and Interpretation, Germany. B. Gottfried (Ed.), 1-16.

8. Spaccapietra, S., Parent, C., Damiani, L., de Macedo, J.A., Porto, F. \& Vangenot, C. (2008). A conceptual view on trajectories. Data Knowledge Engineering, 65, 126-146.

9. Andrienko, G. \& Andrienko N. (2008). Spatio-temporal Aggregation for Visual Analysis of Movements. IEEE Symposium on Visual Analytics Science and Technology (VAST 2008), Columbus, Ohio, USA. IEEE Computer Society Press, 51-58.

10. Dodge, S., Weibel, R. \& Lautenschütz, A.K. (2008). Towards a taxonomy of movement patterns. Information Visualization, 7, 240-252.

11. Frank, L. D., Sallis, J. F., Saelens, B. E., Leary, L., Cain, K., Conway, T. L., \& Hess, P. M. (2010). The development of a walkability index: application to the Neighborhood Quality of Life Study. British journal of sports medicine, 44(13), 924933.

12. King, W. C., Brach, J. S., Belle, S., Killingsworth, R., Fenton, M., \& Kriska, A. M. (2003). The relationship between convenience of destinations and walking levels in older women. American Journal of Health Promotion, 18(1), 74-82.

13. Owen, N., Cerin, E., Leslie, E., Coffee, N., Frank, L. D., Bauman, A. E., Hugo, G., Saelens, B. E., \& Sallis, J. F. (2007). Neighborhood walkability and the walking behavior of Australian adults. American journal of preventive medicine, 33(5), 387395.

14. Saelens, B. E., Sallis, J. F., Black, J. B., \& Chen, D. (2003). Neighborhood-based differences in physical activity: an environment scale evaluation. American journal of public health, 93(9), 1552-1558.

15. Abley, S. (2005). Walkability scoping paper. http://www. levelof service.com/walkability-research.pdf

16. Park, S. (2008). Defining, measuring, and evaluating path walkability, and testing its impacts on transit users' mode choice and walking distance to the station. ProQuest.

17. Transport for London (2005). Improving walkability. http://content.tfl.gov.uk/tfl-improving-walkability.pdf

18. 1000 Friends of Oregon (1993). Making the land use transportation air quality connection-The pedestrian environment. http://ntl.bts.gov/DOCS/tped.html

19. Evans IV, J., Perincherry, V., \& Douglas III, G. (1997). Transit friendliness factor: approach to quantifying transit access environment in a transportation planning model. Transportation Research Record: Journal of the Transportation Research Board, (1604), 32-39

20. Emery, J., Crump, C., \& Bors, P. (2003). Reliability and validity of two instruments designed to assess the walking and bicycling suitability of sidewalks and roads. American Journal of Health Promotion, 18(1), 38-46.

21. Boarnet, M. G., Day, K., Alfonzo, M., Forsyth, A., \& Oakes, M. (2006). The Irvine-Minnesota inventory to measure built environments: reliability tests. American journal of preventive medicine, 30(2), 153-159.

22. Day, K., Boarnet, M., Alfonzo, M., \& Forsyth, A. (2006). The Irvine-Minnesota inventory to measure built environments: development. American journal of preventive medicine, 30(2), 144-152. 
23. Ewing, R., Handy, S., Brownson, R. C., Clemente, O., \& Winston, E. (2006). Identifying and measuring urban design qualities related to walkability. Journal of Physical Activity \& Health, 3, S223.

24. Ewing, R., \& Handy, S. (2009). Measuring the unmeasurable: urban design qualities related to walkability. Journal of Urban design, 14(1), 65-84.

25. Transport for London (2010). Pedestrian comfort guidance for London. https://www . centro.org.uk/media/424876/Appendix-8.PDF

26. Gehl, J., \& Gemzøe, L. (2004). Public spaces-public life (New York: Van Nostrand Reinhold).

27. McArdle, G., Demsar, U., van der Spek, S. \& McLoone, S. (2014). Classifying pedestrian movement behaviour from GPS trajectories using visualization and clustering. Annals of GIS, 20(2), 85-98

28. Wood,. Z. (2014). What can spatial collectives tell us about their environment? IEEE Symposium on Computational Intelligence and Data Mining (CIDM), 2014. Orlando, FL. 329 - 336.

29. Wang, J., Duckham, M. \& Worboys, M. (2015). A framework for models of movement in geographic space. International Journal of Geographic Information Science, DOI:10.1080/13658816.2015.1078466.

30. Schadschneider, A., Kirchner, A. \& Nishinari, K. (2002). CA Approach to Collective Phenomena in Pedestrian Dynamics. In Proceedings of the 5th International Conference on Cellular Automata for Research and Industry, ACRI 2002 Geneva, Switzerland, October 9. 239-248.

31. Freeman, L. C. (1977). A set of measures of centrality based on betweenness. Sociometry, 35-41.

32. Varoudis, T., Law, S., Karimi, K., Hillier, B., \& Penn, A. (2013). Space Syntax Angular Betweenness Centrality Revisited. In Ninth International Space Syntax Symposium, 57, 1-16.

33. Kazerani, A., \& Winter, S. (2009). Can betweenness centrality explain traffic flow. In Proceedings of the 12th AGILE International Conference on GIS.

34. Crucitti, P., Latora, V., \& Porta, S. (2006). Centrality in networks of urban streets. Chaos: an interdisciplinary journal of nonlinear science, 16(1), 015113.

35. Crucitti, P., Latora, V., \& Porta, S. (2006). Centrality measures in spatial networks of urban streets. Physical Review E, 73(3), 036125.

36. Porta, S., Crucitti, P., \& Latora, V. (2008). Multiple centrality assessment in Parma: a network analysis of paths and open spaces. Urban design International, 13(1), 41-50.

37. Porta, S., Latora, V., Wang, F., Strano, E., Cardillo, A., Scellato, S., \& Messora, R. (2009). Street centrality and densities of retail and services in Bologna, Italy. Environment and Planning B: Planning and design, 36(3), 450-465.

38. Produit, T. (2009). A novel GIS method to determine an urban centrality index applied to the Barcelona metropolitan area. Master dissertation (No. EPFLSTUDENT-175634).

39. Valente, T. W., Coronges, K., Lakon, C., \& Costenbader, E. (2008). How correlated are network centrality measures? Connections (Toronto, Ont.), 28(1), 16.

40. Gross, J. L., Yellen, \& J., Zhang, P. (Eds.) (2013). Handbook of graph theory (second edition). CRC Press. 